OPEN ACCESS

Edited by:

Gilles Benichou,

Harvard Medical School,

United States

Reviewed by:

Nuala Mooney,

Centre National de la Recherche

Scientifique (CNRS), France

Frederic Baron,

University of Liège, Belgium

*Correspondence:

José L. Cohen

jose.cohen@inserm.fr

${ }^{\dagger}$ These authors share last authorship

Specialty section:

This article was submitted to Alloimmunity and Transplantation,

a section of the journal

Frontiers in Immunology

Received: 12 July 2019 Accepted: 26 November 2019 Published: 18 December 2019

Citation:

Pilon C, Stehlé T, Beldi-Ferchiou A,

Matignon $M$, Thiolat A, Burlion A, Grondin $C$, Birebent B, Pirenne $F$,

Rouard H, Lang P, Marodon G,

Grimbert $P$ and Cohen JL (2019)

Human Apoptotic Cells, Generated by

Extracorporeal Photopheresis,

Modulate Allogeneic Immune

Response. Front. Immunol. 10:2908.

doi: 10.3389/fimmu.2019.02908

\section{Human Apoptotic Cells, Generated by Extracorporeal Photopheresis, Modulate Allogeneic Immune Response}

\author{
Caroline Pilon 1,2,3, Thomas Stehlé ${ }^{1,2,4}$, Asma Beldi-Ferchiou ${ }^{2,3}$, Marie Matignon 1,2,3,4, \\ Allan Thiolat ${ }^{2,3}$, Aude Burlion ${ }^{5}$, Cynthia Grondin ${ }^{1,2,3}$, Brigitte Birebent ${ }^{6}$, France Pirenne ${ }^{6,7}$, \\ Hélène Rouard ${ }^{6}$, Philippe Lang ${ }^{2,3,4}$, Gilles Marodon ${ }^{5}$, Philippe Grimbert ${ }^{1,2,3,4 t}$ and \\ José L. Cohen ${ }^{1,2,3 * t}$ \\ ${ }^{1}$ Assistance Publique-Hôpitaux de Paris (AP-HP), Groupe Hospitalo-Universitaire Chenevier Mondor, Centre d'Investigation \\ Clinique Biothérapie, Créteil, France, ${ }^{2}$ Institut Mondor de recherche biomédicale, Université Paris-Est, UMR_S955, UPEC, \\ Créteil, France, ${ }^{3}$ Inserm, U955, Equipe 21, Créteil, France, ${ }^{4}$ AP-HP, Groupe Hospitalo-Universitaire Chenevier Mondor, \\ Service de Néphrologie-Transplantation, Créteil, France, ${ }^{5}$ Sorbonne Universités, UPMC Univ Paris 06, INSERM, CNRS, \\ Centre d'Immunologie et des Maladies Infectieuses (CIMI-Paris), Paris, France, ${ }^{6}$ Etablissement Français du Sang (EFS) - Ile \\ de France, Créteil, France, ${ }^{7}$ Inserm, U955, Equipe 2, Créteil, France
}

The induction of specific and sustainable tolerance is a challenging issue in organ transplantation. The discovery of the immunosuppressive properties of apoptotic cells in animal models has paved the way for their use in human transplantation. In this work, we aimed to define a stable, reproducible, and clinically compatible production procedure of human apoptotic cells (Apo-cells). Using a clinically approved extracorporeal photopheresis technique, we have produced and characterized phenotypically and functionally human apoptotic cells. These Apo-cells have immunosuppressive properties proved in vitro and in vivo in $\mathrm{NOD} / \mathrm{SCID} / \gamma \mathrm{C}$ mice by their capacity to modulate an allogeneic response following both a direct and an indirect antigen presentation. These results brought the rationale for the use of Apo-cells in tolerance induction protocol for organ transplantation.

Keywords: tolerance, NSG mice, immunomodulation, transplantation, apoptotic cell

\section{INTRODUCTION}

Organ transplantation has become a successful therapeutic strategy for the treatment of many endstage organ failures. It requires, however, long-term immunosuppressive treatment to overcome the allogeneic immune response, and that in turn is associated with toxicity and high incidence of malignancies, infections, and metabolic diseases. One of the ultimate goals for researchers is to develop novel transplantation therapeutic approaches that induce donor-specific tolerance. By controlling allogeneic recognition, responsible for acute and chronic allograft rejection, it would be possible to lessen the generalized immunosuppression and its associated side effects.

Extracorporeal photopheresis (ECP) appears as a promising strategy to achieve the desired goal. ECP is a therapeutic regimen approved as a palliative treatment for the skin manifestations associated with cutaneous T-cell lymphoma (1). In transplantation, ECP has also been proven effective in a variety of clinical indications including solid organ transplantation (2) and hematopoietic stem cell transplantation (3). ECP can be in some cases an effective approach in 
steroid-resistant chronic graft-vs.-host disease (GvHD), thus sparing patients the glucocorticoid or immunosuppressant therapies (4). Thanks to its efficacy and the few related adverse effects, ECP is highly recommended for the prevention of graft rejection in heart transplantation (5), and can be proposed as a prophylaxis for GvHD in myeloablative HLA-matched allogeneic bone marrow transplantation (6) and in grade I and II acute GvHD (3). On the same clinical setting, a recent study has also shown the interest of combining ECP with other immune-based approaches like low-dose IL-2 (7).

Despite its therapeutic interest and wide-range coverage, the mechanisms of action of ECP are poorly known and sometimes contradictory. Surprisingly, it was reported that ECP promoted the release of prototypic immune-stimulatory cytokine IL-1 $\beta$ (8) and that the treated monocytes retained their ability to differentiate into fully functional dendritic cell (DC) that maturated and stimulated $\mathrm{T}$ cells as well as normal DC (9). On the other hand, a bunch of arguments, built on data collected mainly on rodents, strongly suggest that ECP-induced immunomodulation could be mediated largely by apoptosis resulting from exposure of mononuclear cells to UV-A or UV-B. Apoptotic cells have the ability to downregulate the inflammatory and the immune responses by delivering inhibitory signals to phagocytes $(10,11)$, particularly DCs (12). The latter internalize cells in early apoptosis; exhibit a selective decrease in the levels of pro-inflammatory cytokines IL-1a, IL-1b, IL-6, IL-12p70, and TNF- $\alpha$; and secret normal or increased amounts of immunosuppressive TGF$\beta$ and IL-10 (13-15). Given the efficient capacity of DC to internalize apoptotic cells (16) making it a key player in peripheral T-cell tolerance (17), direct administration of donor apoptotic cells could be envisaged to simultaneously deliver both inhibitory signals and donor allogeneic antigens to recipient DC. For such, a study has shown that intravenous administration of donor apoptotic cells is an alternative method to prevent DC activation and to present donor allogeneic antigen to recipient DC leading to a significant control of the anti-donor response in a rat model of heart transplantation (18). Such results open new perspectives for donor-specific tolerance induction in solid organ transplantation in human.

Although preclinical results are robust in animal models of transplantation, data on the characterization and immunosuppressive properties of apoptotic cells obtained from human samples remain rare. In vitro, it was suggested that 8-MOP-treated and UV-A-irradiated cells could acquire a partial immunogenic phenotype that triggers phagocytosis of apoptotic cells by macrophages and DC; however, it was not enough to induce DC maturation and T-cell activation (19). In the present study, we used apoptotic cells generated from human peripheral blood mononuclear cells (PBMCs) that were incubated with 8-MOP and exposed to UV-A radiation (referred to as Apo-cells); the procedure and the tools are clinically validated. After phenotypic characterization, induction of primary and secondary mixed lymphocyte reaction (MLR), and in vivo testing of allogeneic immune responses, we demonstrate for the first time the capacity of human Apo-cells, obtained by
ECP, to down-regulate allogeneic T-cell response. This work opens new therapeutic perspectives for allograft management.

\section{MATERIALS AND METHODS}

\section{Blood Samples}

Human PBMCs were obtained from unrelated and unmatched healthy donors (Etablissement Francais du Sang, Créteil, France). PBMCs were isolated by density gradient centrifugation (Lymphocyte Separation Medium; Eurobio ${ }^{\circledR}$, France), and the cells were suspended in RPMI-1640 + GluMAX (Life Technologies, France) supplemented with penicillin/streptomycin (Life Technologies, France) and 10\% fetal bovine serum (Life Technologies, France).

\section{Induction and Labeling of Apoptotic Cells}

Apoptosis of PBMC was induced by UV-A irradiation after 8-MOP (Methoxsalene, Macopharma) sensitization, as follows: PBMCs were transferred to a bag specially adapted for UVA irradiation (Macopharma, Mouvaux, France), and $200 \mathrm{ng} / \mathrm{ml}$ of 8-MOP was added for $15 \mathrm{~min}$ at $37^{\circ} \mathrm{C}$ before $\mathrm{UV}$ A irradiation at $2-4 \mathrm{~J} / \mathrm{cm}^{2}$ (Vilbert-Lourmat, Bio-Génic, or Macogenic, Macopharma). Treated cells were then incubated at $37^{\circ} \mathrm{C}$. After a 16 to $18 \mathrm{~h}$ incubation, UV-treated cells were incubated with 7AAD (BD Biosciences) for $15 \mathrm{~min}$ at room temperature, and then DiOC6 $(67 \mu \mathrm{M})$ was added and cells were incubated again for $15 \mathrm{~min}$ at $37^{\circ} \mathrm{C}$ before flow cytometry analysis. DiOC6/7AAD (3,3-dihexyloxacarbocyanine Iodide/7-amino-actinomycinD) staining dyes were used to quantify apoptosis and necrosis. DiOC6 (Molecular Probes ${ }^{\circledR}$; Life Technologies ${ }^{\mathrm{TM}}$, ref D-273) was used to monitor potential mitochondrial trans-membrane disruption.

\section{Allogeneic MLR: Primary and Secondary}

The responding cells (PBMCs) were first CFSE-labeled [5(6)-carboxyfluorescein diacetate N-succinimidyl ester; SigmaAldrich] and incubated at a ratio 1:1 (concentration of $1 \times 10^{6}$ cells/ml) with stimulating allogeneic cells [Apo-cells or gammairradiated (20 Gy) PBMCs] at $37^{\circ} \mathrm{C}$ for 5 days. As a control, non-stimulated responding cells were cultivated in medium alone. The primary MLR was initiated by cultivating CD2cells (CFSE-labeled), obtained from stimulating donor cells or non-labeled apoptotic cells, with allogeneic responding cells for 5 days. For the secondary stimulation, CD4-CD8 living cells from responding donor $\left(\mathrm{CD}^{+}, \mathrm{CD}^{+}, \mathrm{CFSE}^{-}, 7 \mathrm{AAD}^{-}\right)$were sorted by fluorescence-activated cell sorting (FACS) (MoFlo Legacy, Beckman Coulter), then stained with cell proliferation dye eFluor450 (eBiosciences, France), and cultured with initial PBMCs from stimulating donor or third-party PBMCs at a ratio $1: 1$.

\section{Autologous MLR}

Antigen-presenting cells (APCs) (CD2-) were first separated from T cells and NK cells by CD2 isolation. CD2+ was obtained by magnetic selection using Miltenyi technology (Miltenyi Biotec). PBMCs were first incubated with corresponding microbeads (anti-CD2) for $15 \mathrm{~min}$ and enriched in desired 
cells upon passage through large magnetic selection columns, according to the manufacturer's instructions. The obtained APCs (CD2-) were cultivated with allogeneic Apo-cells or control PBMCs at a ratio 1:1 for $48 \mathrm{~h}$. Phenotypic analysis was then performed. For autologous MLR, the $48 \mathrm{~h}$ treated APCs were washed and then cultured with their own, previously isolated, CFSE-labeled CD2+ cells and kept at $37^{\circ} \mathrm{C}$ for $48 \mathrm{~h}$ at a ratio $1: 1$ for another 5 days.

\section{Cytokine Production}

The amount of human IFN $\gamma$, IL- 6 , and TNF- $\alpha$ in the culture supernatant was determined using Duoset ELISA (R\&D Systems) according to the manufacturer's instruction.

\section{mRNA Isolation and Real-Time Quantitative PCR}

mRNA was extracted from $5 \times 10^{6}$ PBMCs or UV-A-treated PBMCs using the RNeasy mini kit (Qiagen, Valencia, CA) according to the manufacturer's instructions. Genomic DNA was removed by DNase treatment (Qiagen). Total RNA was reverse transcribed to complementary DNA using reverse transcription reagents (Thermo Scientific, Courtaboeuf, France). Real-time quantitative PCR was performed using commercially available primer and probe sets (Applied Biosystems): HPRT, Hs99999909_m1; BAX, Hs00180269_m1; BCL2, Hs00608023; CASP3, Hs00234387; IL-10, Hs00961622_m1; and TGF- $\beta 1$, Hs00998133_m1. We used the $2-\Delta \Delta$ ct method to calculate the relative expression of RNAs between a sample and a reference. All samples were tested in duplicates in 96-well plates with 7900HT fast real-time PCR system (Applied Biosystems, Foster City, CA, USA). HPRT was used as endogenous controls to normalize RNA amounts.

\section{Flow Cytometry}

The antibodies used for FACS analysis are summarized in Table 1. The efluor 450-labeled anti-Foxp3 staining was performed using the eBioscience kit and protocol. The fixation/permeabilization kit (BD Biosciences) was used for intra-cellular staining of active caspase-3. Cell proliferation dye 450 (ebioscience) was used to help exclude APCs in the in vivo experiment. Flow cytometry events were acquired on a FACSCanto II flow cytometer (BD Biosciences) and analyzed using FlowJo (Tree Star, Ashland, OR, USA) software.

\section{In vivo Experiment}

Ten- to fourteen-week-old NOD/SCID/ $/ \mathrm{C}$ (NSG) mice, bred in our animal facility under specific pathogen-free conditions, were used for the purpose. All experimental protocols were approved by the local ethical committee (Ce5/2012/025) and are in compliance with the European Union guidelines. For the first infusion, $5 \times 10^{6}$ Apo-cells or $5 \times 10^{6} \mathrm{CD} 2-$ cells (cell proliferation dye-labeled to separate them by flow cytometry) were co-injected intravenously with $5 \times 10^{6}$ allogeneic CD2+ cells. At day $6,0.5 \times 10^{6} \mathrm{CD} 2-$ cells (cell proliferation dye-labeled) from the first donor were injected. 5-Ethynyl-2' deoxyuridine (EdU) was injected (intraperitoneally) at days 10 and 11. At day 11 ( $2 \mathrm{~h}$ after EdU second injection), the mice were sacrificed and their spleens harvested. Percentages of EdU+ cells were assessed using Click-iT ${ }^{\mathrm{TM}}$ EdU Alexa Fluor ${ }^{\mathrm{TM}} 488$ Flow Cytometry Assay Kit (ThermoFisher) according to the manufacturer's protocol.

\section{Statistical Analysis}

Results are expressed as mean \pm SEM for flow cytometry and ELISA analysis or as median [interquartile] for quantitative PCR. We used paired or unpaired Student $t$-test or one-way ANOVA with post-hoc analysis depending on the number of comparatives to calculate $p$-values with Prism 5.0 (Graph Pad Software, Inc., La Jolla, CA, USA). The means were considered significantly different (rejection of the null hypothesis) if $p<0.05$. No a priori statistical testing was performed to check the adequacy of the number of samples with statistical power: ${ }^{*} p<0.05,{ }^{* *} p<0.01$, ${ }^{* * *} p<0.001,{ }^{* * * *} p<0.0001$.

TABLE 1 | Antibodies used for flow cytometry experiments and in vitro assay.

\begin{tabular}{|c|c|c|c|c|c|c|c|}
\hline & Fluorochrome & Clone & Supplier & & Fluorochrome & Clone & Supplier \\
\hline CD3 & PE & UCHT1 & BD biosciences & CD45RO & PerCP & UCHL1 & Miltenyi \\
\hline CD3 & V450 & UCHT1 & BD biosciences & CD56 & APC & B159 & BD biosciences \\
\hline CD4 & APC & RPA-T4 & BD biosciences & CD69 & APC-Су7 & FN50 & BD biosciences \\
\hline CD4 & $\mathrm{PE}$ & RPA-T4 & BD biosciences & CD86 & APC & 2331(FUN-1) & BD biosciences \\
\hline CD4 & APC-Сy7 & RPA-T4 & BD biosciences & CD95 & FITC & DX2 & BD biosciences \\
\hline CD8 & FITC & RPA-T8 & BD biosciences & CD127 & PerCP-Cy5.5 & HIL-7R-M21 & BD biosciences \\
\hline CD8 & $\mathrm{PE}$ & RPA-T8 & BD biosciences & CCR7 & PE-Cy7 & 3D12 & BD biosciences \\
\hline CD8 & PE-Cy7 & RPA-T8 & BD biosciences & Foxp3 & eFluor450 & $\mathrm{PCH} 101$ & eBiosciences \\
\hline CD11c & $\mathrm{PE}$ & B-Ly6 & BD biosciences & HLA-DR & V500 & G46-6 & BD biosciences \\
\hline CD14 & PE-Cy7 & M-A251 & BD biosciences & ICOS & $\mathrm{PE}$ & DX29 & BD biosciences \\
\hline CD19 & V500 & HIB19 & BD biosciences & OX40 & APC & ACT35 & Miltenyi \\
\hline CD25 & PE-Cy7 & $2 \mathrm{~A} 3$ & BD biosciences & TGF $\beta$ & Purified & 1D11.16.8 & Ebiosciences \\
\hline
\end{tabular}




\section{RESULTS}

\section{Exposure of Human PBMCs to UV-A Induces Apoptosis and Phenotypic Modifications}

In order to stay close to a cell product potentially available in humans, we used throughout this study a procedure based on exposing healthy donors' PBMCs to UV-A irradiation after 8-MOP sensitization, a procedure of ECP already in use in clinics. A first step was consisted of characterizing the cellular product and defining the optimal ECP procedure for the generation of strong apoptosis (potentially immunosuppressive) with reduced necrosis (potentially pro-inflammatory). After different incubation periods that followed for UV-A exposure, DiOC6/7AAD staining was performed to discriminate necrosis $\left(7 \mathrm{AAD}^{+} / \mathrm{DiOC}^{-}\right)$from apoptosis $\left(7 \mathrm{AAD}^{-} / \mathrm{DiOC6}^{-}\right)$. We observed that the larger cells, of mainly monocytes, are more sensitive to ECP than lymphocytes as attested by the forward scatter and side scatter parameters. The lower rates of necrotic cells in lymphocytes and monocytes (4.52 $\pm 0.47 \%$ and $29.19 \pm 3.27 \%$, respectively) and the higher rates of apoptotic cells in the same cell populations (41.52 $\pm 2.43 \%$ and $68.64 \pm 3.44 \%$, respectively) were obtained after $16 \mathrm{~h}$ of incubation (Figure 1A) as compared with shorter or longer periods of incubation (data not shown). Concomitantly, the levels of TGF- $\beta$ and IL-10 mRNA were remarkably increased

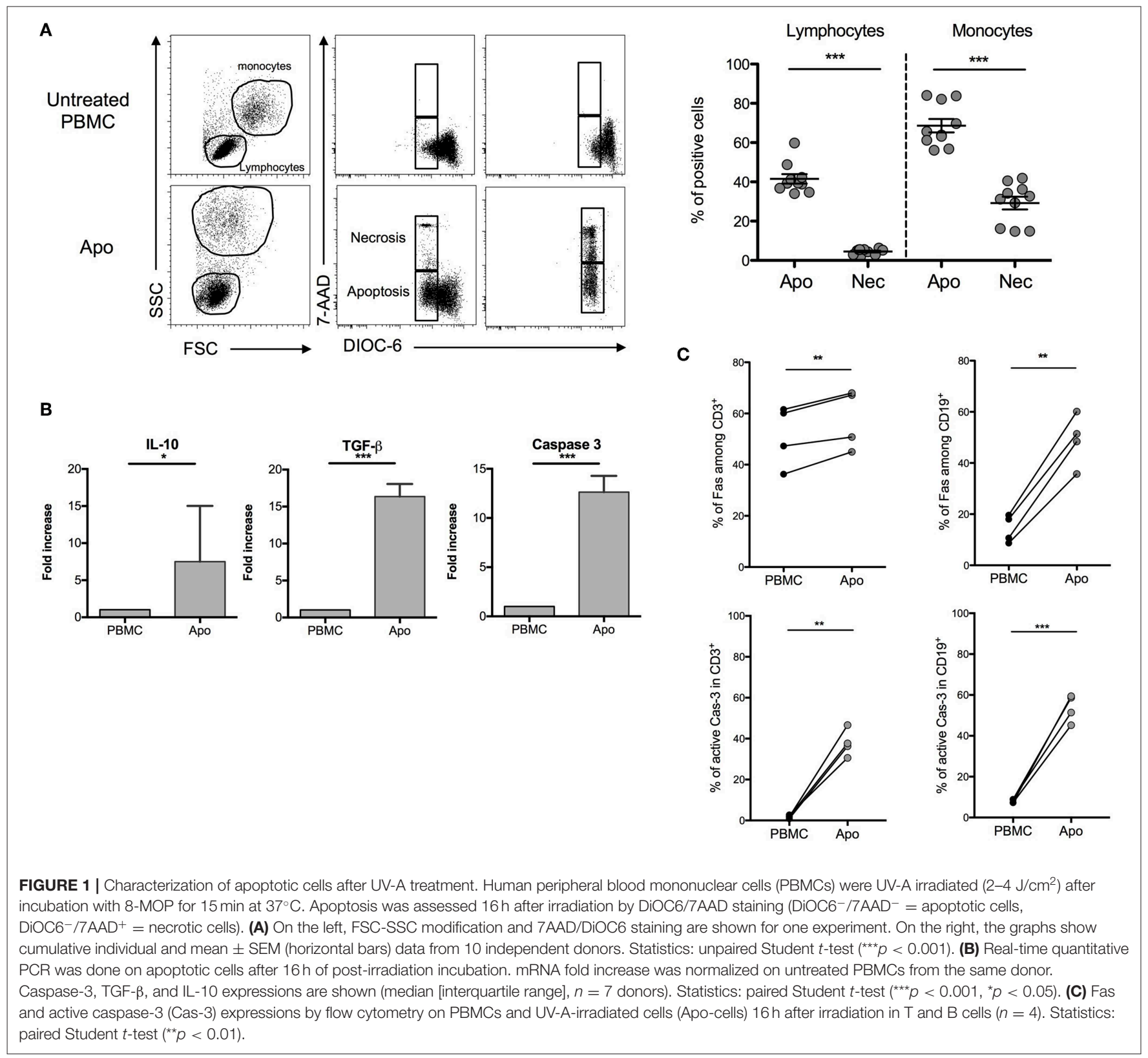


in ECP-treated cells (also called Apo-cells), suggesting a potential shift to an immunosuppressive profile generated by 8-MOP sensitization and UV-A exposure (Figure 1B). The increased expression level of caspase-3 mRNA (involved in early apoptotic cascade) observed in Apo-cells as compared with PBMCs suggests apoptosis induction after UV-A irradiation (Figure 1B), as well as Fas protein expression both in $\mathrm{T}$ and $B$ cells (Figure 1C). Caspase-3 mRNA up-regulation has been reported as an indicator of TCR activation independently of caspase-3 activity and the induction of apoptosis (20). In our work, the percentage of active caspase- 3 was also dramatically increased in both Apo-CD3 and Apo-CD19 cells (Figure 1C), which reinforces the notion of apoptosis triggered under these experimental conditions. Thus, we defined the " $16 \mathrm{~h}$ of post-UVA exposure incubation at $37^{\circ} \mathrm{C}$ " as the optimal procedure to generate Apo-cells.

When we looked at the relative proportions of $\mathrm{T}, \mathrm{NK}$, and $\mathrm{B}$ cells, we could not detect any substantial changes (Figure 2A).
However, the expression of MHC class II molecules (HLADR) on monocytes and B-lymphocytes decreased in Apo-cells, suggesting decreased antigen presentation capacity in these cell populations (Figure 2B). CD4+ T cells in PBMCs did not express CD69, whereas we could detect $5-20 \%$ of CD4+CD69+ T cells in Apo-cells. In contrast, the subpopulation of CD $4+C D 25+T$ cells dropped from $4-6 \%$ to a residual rate in Apo-cells in consistency with the disappearance of CD4+CD127-Foxp3+ subpopulation of T-reg (Figure 2C).

\section{Human Apoptotic Cells Induce Hypo-Responsiveness of Allogeneic Lymphocytes by Both Direct and Indirect Presentation Pathways}

We wanted to establish the ability of Apo-cells to generate allogeneic response in vitro. In order to imitate the mechanism of direct alloantigen presentation, Apo-cells were incubated

A

\begin{tabular}{ccccccc}
\cline { 2 - 6 } & CD3 (\%) & CD4 (\%) & CD8 (\%) & B cells (\%) & NK cells (\%) & Myeloid (\%) \\
\hline PBMC & $73.96 \pm 3.52$ & $48.7 \pm 3.34$ & $22.88 \pm 3.94$ & $10.54 \pm 1.96$ & $10.26 \pm 1.70$ & $8.07 \pm 1.94$ \\
Apo & $73.38 \pm 3.58$ & $41.40 \pm 2.94$ & $26.84 \pm 4.22$ & $13.64 \pm 1.19$ & $11.39 \pm 1.51$ & $14.30 \pm 0.98$ *
\end{tabular}

B
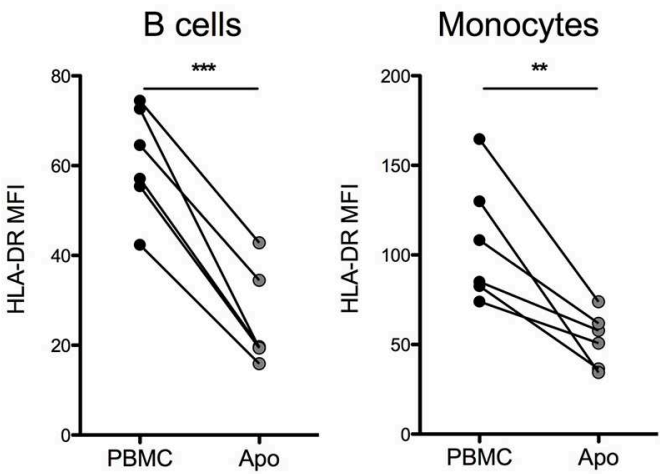

C
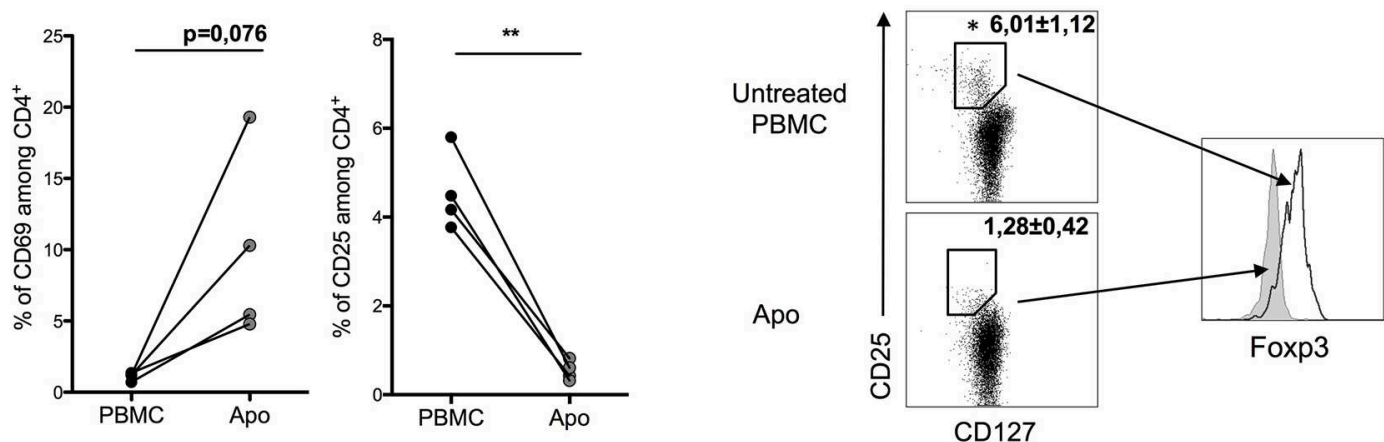

FIGURE 2 | Effect of UV-A treatment on lymphocytes population. Untreated PBMCs and UV-A-irradiated cells (Apo-cells) from the same donor were phenotypically characterized after $16 \mathrm{~h}$ of incubation. (A) Lymphocytes populations were analyzed and surface-stained using CD3+ CD4+/CD8 for T cells, CD19+ for B cells, and CD56 + for NK cells and myeloid cells based on FSC-SSC size (mean \pm SEM) $n=4-6$ independent experiments. (B) HLA-DR mean fluorescence intensity (MFI) was assessed on B cells and monocytes ( $n=6$ independent experiments). Statistics: paired Student $t$-test $\left({ }^{*} p<0.05,{ }^{* *} p<0.01,{ }^{* * *} p<0.001\right)$. (C) Activation markers CD25 and CD69 were analyzed on CD4 T cells; T-reg population was described by activation markers CD127 $7^{\text {low }} \mathrm{CD}^{2} 5^{\text {high }}$ Foxp3 ${ }^{+}(n=3-4$ independent experiments). Statistics: paired Student $t$-test $\left({ }^{\star} p<0.05,{ }^{* \star} p<0.01\right)$. 


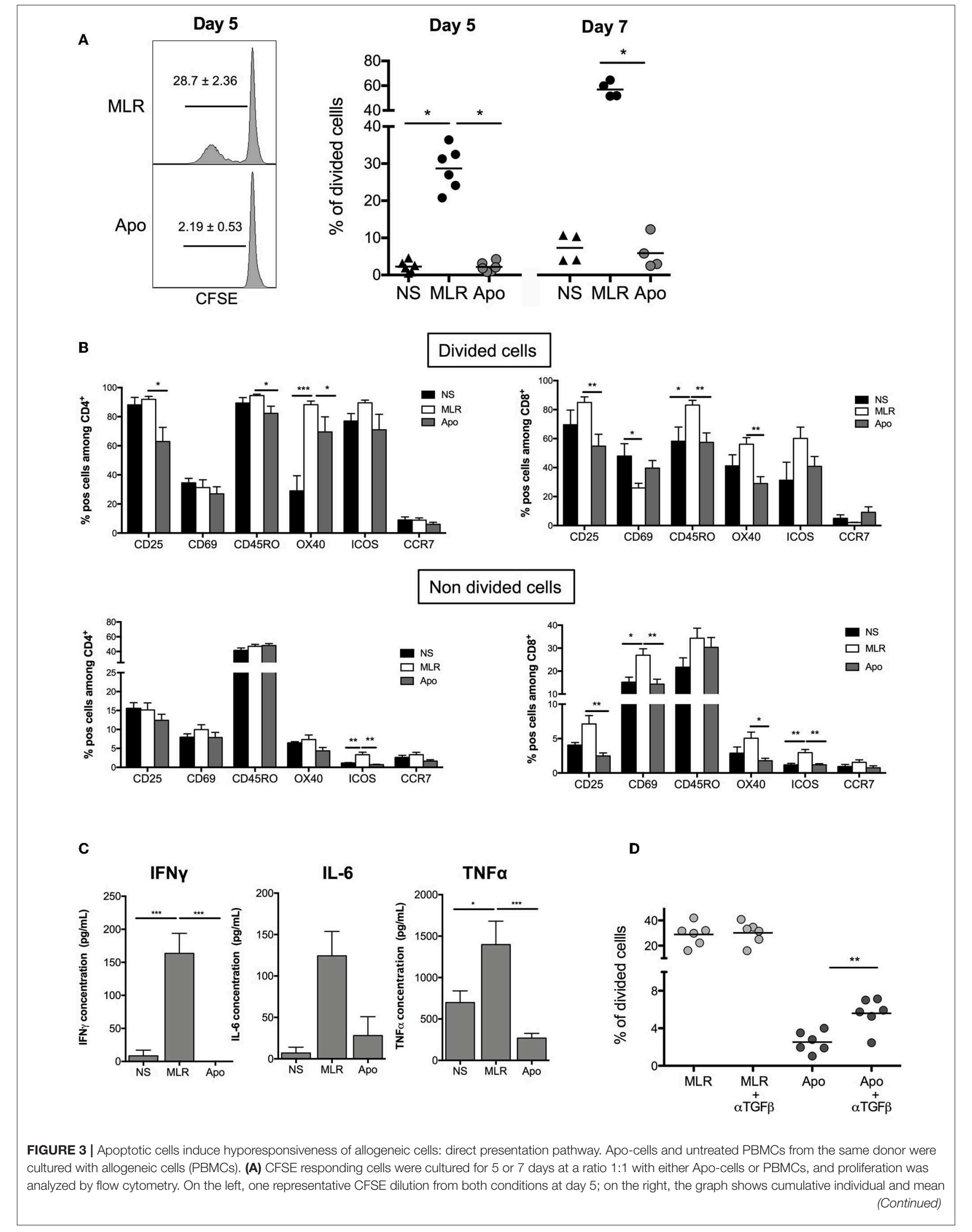


FIGURE 3 | \pm SEM (horizontal bars) data from four to six independent experiments for day 5 and day 7 . Statistics: one-way ANOVA with post-hoc $\left({ }^{*} p<0.05\right)$. (B) Results are presented as mean \pm SEM of the percentages of activation markers CD25 ${ }^{+}, \mathrm{CD} 69^{+}, \mathrm{CD} 45 \mathrm{RO}^{+}, \mathrm{OX}^{+} 0^{+}, \mathrm{ICOS}^{+}, \mathrm{and}_{\mathrm{CCR}}{ }^{+}$cells on allostimulated $\mathrm{T}$ cells and on non-stimulated T cells (NS) at day 5 for divided cells (up) and non-divided cells (down) for CD4 ${ }^{+}$cells (left) and CD8 ${ }^{+}$cells (right). (C) Results are presented as mean \pm SEM of supernatant concentrations of IFN $\gamma$, IL-6, and TNF- $\alpha$ cytokine analyzed by ELISA at day 5 . Graphs show cumulative date of four to six independent experiments. Statistics: one-way ANOVA with post-hoc ( $\left.{ }^{\star} p<0.05,{ }^{* *} p<0.01,{ }^{* * *} p<0.001\right)$. (D) CFSE responding cells were cultured for 5 days at a ratio of $1: 1$ with or without TGF- $\beta$ neutralizing antibody, and proliferation was analyzed by flow cytometry. Percentages of divided cells after 5 days of co-culture.

Graphs show cumulative individual data from six independent experiments. Statistics: unpaired Student $t$-test $\left({ }^{* \star} p<0.01\right)$.

with allogeneic PBMCs. In the MLR groups, we used $\gamma$ irradiated cells to activate allogeneic PBMCs. In such a setting, $\gamma$-irradiation could also induce apoptosis. However, apoptosis/necrosis profile strongly differed notably for monocytes (Supplemental Figure 1) as compared with Apo-cells (Figure 1). After 5 days of co-culture, Apo-cells did not induce proliferation of allogeneic PBMCs as attested by CFSE dilution compared with standard MLR using untreated irradiated PBMCs from the same donor as stimulating cells $(2.20 \pm 0.53 \%$ vs. $28.68 \pm 2.36 \%$, respectively; Figure 3A). The low proliferation rate induced by Apo-cells does not seem to be related to the response time since the differences from irradiated PBMCs were even more pronounced when the culture was prolonged to day 7 (56.93 $\pm 3.17 \%$ for PBMCs vs. $5.88 \pm 2.26 \%$ for Apo-cells). In addition to the decreased proliferation of responding cells in the presence of Apo-cells, T-cell activation markers (CD25, CD69, OX40, and ICOS) as well as memory phenotype markers (CD45RO and CCR7) were analyzed (Figure 3B). In the divided cells, we observed a statistically significant reduction in the proportion of CD4+ T cells expressing CD25 and in CD8+ T cells expressing CD25 and OX40, with a trend to a decrease in ICOS in Apo-cells as compared with irradiated PBMCs. The activated/memory marker CD45RO decreased in both CD4 and CD8, whereas there was no significant change in CCR7 expression. In the non-divided cells, significant changes of expression were observed mainly in CD8 population with a decrease in CD25, CD69, and OX40 and a trend to a decrease in ICOS expression in Apo-cells compared with irradiated PBMCs. In CD4 cells, only ICOS and CCR7 decreased (Figure 3B). The low priming capacity of Apo-cells was also reflected via the poor secretion of inflammatory cytokines such as IFN $\gamma$, IL-6, and TNF- $\alpha$ by responding cells, as compared with cells cultured with irradiated PBMCs (Figure 3C).

It was previously shown that apoptotic cells may exert their suppressive activity by secreting TGF- $\beta$ (10). In our hands, the level of mRNA TGF- $\beta$ was elevated in Apo-cells (Figure 1B). Interestingly, when we quantified TGF- $\beta$ concentration in the culture at day 5 , we did not observe any difference upon using irradiated PBMCs or Apo-cells (data not shown). By then, we reproduced MLR experiments in the presence of anti-TGF- $\beta$. In this setting, Apo-cell stimulatory capacity was partially restored (Figure 3D), suggesting that TGF- $\beta$ produced in the presence of apoptotic cells might be partly responsible for the poor response of allogeneic responding cells. Blocking IL-10 had no effect on the culture (data not shown).

In order to evaluate the role of Apo-cells in the indirect presentation of alloantigens, we first incubated APCs (CD2cells, containing mainly $\mathrm{B}$ cells and monocytes) with allogeneic
Apo-cells or untreated irradiated allogeneic PBMCs from the same donor and studied the impact on the activation of APC (Figure 4A). After $48 \mathrm{~h}$ of co-culture of allogeneic Apocells with CD2- cells, the percentage of CD14+CD11c+ myeloid cells increased as compared with co-culture with untreated PBMCs $(18.35 \pm 2.9 \%$ vs. $7.28 \pm 2.92 \%$, respectively; Figure 4B). When we looked at HLA-class II expression on CD14+CD11c+, CD14-CD11c+, and CD19+ cells, in all the HLA-DR, mean fluorescence intensity was statistically decreased when CD2- cells were incubated with Apo-cells as compared with irradiated PBMCs (Figure 4C). CD86 is expressed on APC and known to provide costimulatory signals required for T-cell activation and survival. In the presence of allogeneic Apo-cells, the mean fluorescence intensity of CD86 expression on CD14-CD11c+ DCs and CD19 B cells was decreased compared with CD2- cells incubated with allogeneic PBMCs (Figure 4C). In alignment, co-incubation of CD2- with Apo-cells induced a dramatically reduced production of pro-inflammatory cytokines IL- 6 and TNF- $\alpha$ compared with co-incubation with irradiated PBMCs (Figure 4D). The ability of pre-incubated CD2- cells to induce proliferation of autologous lymphocytes was analyzed. CD2- cells sorted out at day 0 were cultured from day 2 with CSFE-labeled autologous CD2+ cells for 5 days. Under such conditions, $10 \%$ of $\mathrm{T}$ cells underwent at least one division cycle at day 7 . When CD2- cells were preincubated for 2 days in the presence of allogeneic irradiated PBMCs and then cultured for 5 days with CSFE-labeled autologous CD2+ cells, the percentage of divided $\mathrm{T}$ cells increased to reach $23.42 \pm 7.56 \%$. When allogeneic irradiated PBMCs were replaced by allogeneic Apo-cells, the percentage of divided $\mathrm{T}$ cells dropped to $6.32 \pm 1.56 \%$ (Figure $4 \mathrm{E}$ ). Altogether, these results demonstrate the low allogeneic priming capacity of human Apo-cells both in the direct and indirect presentation pathways.

\section{Apo-Cells as a Tool to Modulate Allogeneic Immune Response}

Our aim was to assess the possibility of using allogeneic Apo-cells as a strategy of tolerance induction in transplantation. In vitro, we developed a procedure consisting of alloantigen sensitization followed by a step of recall with the same antigen, thus simulating organ transplantation consecutive to Apo-cell administration. For such, PBMCs were cultured for 5 days in the presence of either allogeneic Apo-cells or irradiated PBMCs. Then, T cells were sorted out using flow cytometry, CFSE-labeled, and cultured in the presence of allogeneic irradiated PBMCs from the same donor (Figure 5A, top). After 5 days of culture, $18.47 \pm 2.28 \%$ of $\mathrm{T}$ cells cultured with allogeneic PBMCs had divided at least 
A

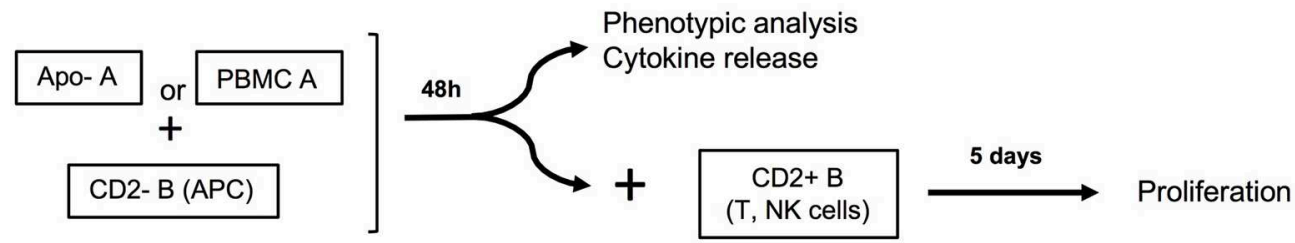

B
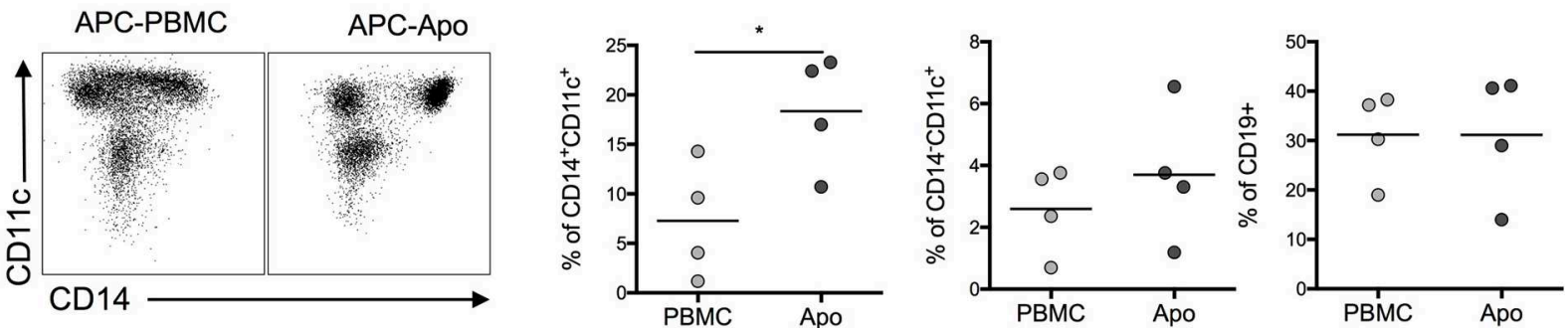

C

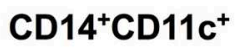

CD14-CD11c ${ }^{+}$

B cells
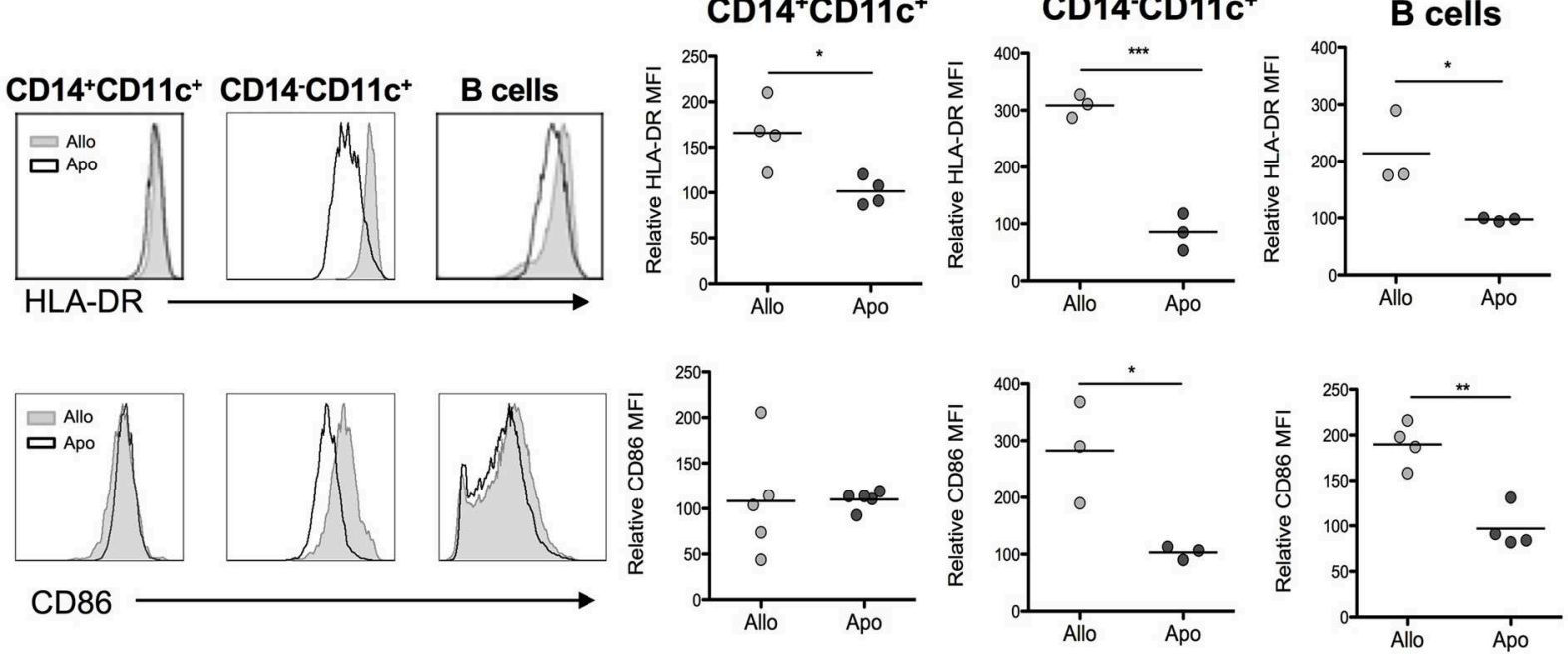

D

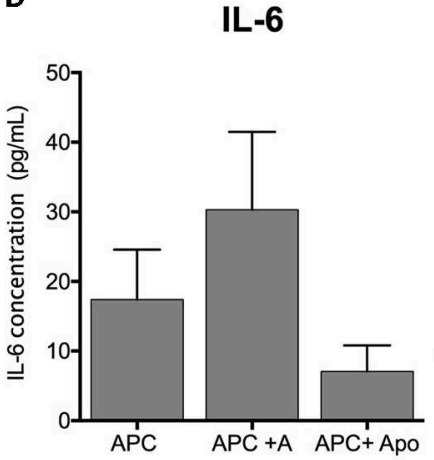

TNF $\alpha$

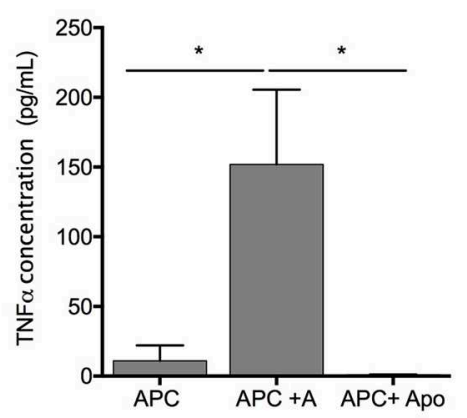

E
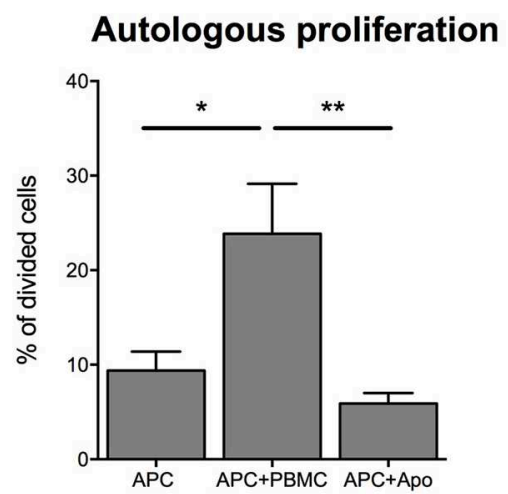

FIGURE 4 | Apoptotic cells induce hyporesponse of allogeneic cells: indirect presentation pathway. (A) Experimental scheme. APCs (CD2- cells) were incubated with allogeneic Apo-cells or untreated irradiated-allogeneic PBMCs for $48 \mathrm{~h}$. Phenotypic analysis was done on APCs, which were then used to stimulate autologous, CFSE-stained CD2+ cells. (B) Flow cytometry analysis of APCs showing the ratio of CD14+CD11c-, CD14+CD11c, and CD19+ cells. (C) Left: overlay comparison 
FIGURE 4 | of HLA-DR and CD86 expression of allo and Apo-cells. Right: HLA-DR and CD86 relative MFI (normalized on the expression on APCs cultivated alone for $48 \mathrm{~h}$ ) ( $n=3-4$ independent experiments). Statistics: unpaired Student $t$-test $\left({ }^{*} p<0.05,{ }^{* *} p<0.01,{ }^{* *} p<0.001\right)$. (D) Results are presented as mean \pm SEM of supernatant concentration of IL- 6 and TNF- $\alpha$ cytokine analyzed by ELISA after $48 \mathrm{~h}$. Graphs show cumulative data of four independent experiments. Statistic: one-way ANOVA with post-hoc ( $\left.{ }^{*} p<0.05\right)$. (E) CFSE autologous responding cells were cultured for 5 days at a ratio $1: 1$ with APCs, APCs + PBMCs, or APCs + Apo-cells, and proliferation was analyzed by flow cytometry; cumulative individual and mean \pm SEM (horizontal bars) data from five independent experiments. Statistics: 1-way ANOVA with post-hoc $\left({ }^{\star} p<0.05,{ }^{\star \star} p<0.01\right)$.

once. After recall antigen stimulation, divided cells represented $27.68 \pm 6.6 \%$ of sorted out T cells. When the first stimulation was performed with allogeneic Apo-cells, we could not detect any division, as we saw in Figure 3A. After priming PBMCs with Apo-cells and having sorted out $\mathrm{T}$ cells cultured in the presence of irradiated allogeneic PBMCs, only $3.75 \pm 1.14 \%$ of $\mathrm{T}$ cells divided, a percentage statistically inferior to that observed in the control group (27.68 $\pm 6.6 \%)$, and even lower than that observed with PBMCs after the first simulation with allogeneic irradiated PBMCs (Figure 5A). Upon culturing sorted-out $\mathrm{T}$ cells in the presence of third-party irradiated PBMCs, we detected a slight but not statistically significant difference from the Apo-A/Allo-A group, suggesting that the mechanism of suppression exerted by Apo-cells was partly Ag-specific. We then worked to reproduce this observation in vivo in immune-compromised NSG mice that sustain massive proliferation of human $\mathrm{T}$ cells following xeno-GvHD (21-23). Precisely, we wanted to investigate whether Apo-cells could modify human T-cell expansion in vivo. The first step of sensitization consisted of co-injecting NSG mice with either allogeneic CD2- cells or allogeneic Apo-cells with $\mathrm{CD} 2+$ cells (containing mainly $\mathrm{T}$ and $\mathrm{NK}$ cells). Five days later, mice received a second dose of allogeneic CD2- cells from the same source used for the first stimulation (Figure 5B, top). By then, spleen cells of NSG mice were collected and showed two main features. First, the percentages of $\mathrm{CD} 3+$ cells collected from mice sensitized with allogeneic Apo-cells were dramatically lower than those from mice sensitized with CD2- cells, in consistency with the reduced number of CD3+ cells. We reproduced the same experiment starting with Apocells or APCs autologous to CD2+. When the second in vivo stimulation was performed with allogeneic APCs, we observed a strong decrease in the percentage and number of $\mathrm{CD} 3+$ cells, similar to that obtained when starting with allogeneic cells (Supplemental Figure 2). Second, the CD4/CD8 cell ratio was also dramatically modified to the benefit of $\mathrm{CD} 4+$ cells in mice sensitized with Apo-cells as compared with CD2- cellsensitized mice. The increase in CD4+ T cell percentage was not related to Treg increase (data not shown). We also compared the rate of T-cell division in relationship with the strategy of sensitization, and for such, mice were treated with EdU from day 10 to day 11 . We could not detect any statistical difference between CD4 and CD8 T-cell divisions. The slight increase in EdU incorporation observed in mice initially stimulated by Apo-cells could be attributed to the increased lymphopeniainduced proliferation, which had resulted from the reduction in numbers of $\mathrm{T}$ cells detected in the spleen of this group of mice (Figure 5B, down).

\section{DISCUSSION}

Inducing donor-specific immunosuppression or long-term tolerance in transplantation has been a challenging hypothesis for several decades fed by clinical observations in transplanted patients combined with widened knowledge on the immune system, and particularly the immunosuppressive role of Apocells. A strong rationale came first from kidney-transplanted patients who used to be poly-transfused in order to treat their chronic renal dysfunction-induced anemia. Some of them seemed to have fewer rejection manifestations, while others developed enhanced anti-HLA antibodies with deleterious effects on the outcome of the graft. Such observations have remained unexplained both clinically and in terms of the potentially involved immune mechanisms. The emergence of living donor transplantation incited researchers to develop donor-specific transfusion, which may improve long-term graft survival through specific education of the recipient's cellular immune system toward donor antigens (24). However, solid clinical evidence demonstrated by a randomized clinical trial is still missing, and overall, the immunological mechanisms supporting a tolerance-gaining effect via donor-specific transfusions are still misunderstood. The more recent understanding of the immune modulatory effect of Apo-cells, present in transfusion cellular products, could enlighten our comprehension of a pre-transplant transfusion benefit. Particularly, it could help to develop specific tolerance induction strategies in solid organ transplantation. There is a fairly abundant literature demonstrating the immunosuppressive effect of Apo-cells and the possibility of using them to induce tolerance in transplantation. Most of these studies were conducted on animal models $(13,18,25-30)$. However, the possibility of generating reproducible and clinically compatible apoptotic cells from blood samples in humans has not been described yet, and very little is known about the immune-modulatory properties of human apoptotic cells $(10,11,31)$.

In this study, we first focused on generating and characterizing human Apo-cells by developing a protocol compatible with clinical practice. For this purpose, we started with peripheral blood cells incubated with 8-MOP and exposed to UV-A. This first step allowed us to define stable and reproducible conditions to produce a cellular base of $\mathrm{T}$ and monocyte cells, rich in apoptotic cells and poor in necrotic cells. These cells produce IL-10 and TGF- $\beta$, express caspase- 3 and Fas, and have an apoptotic immunosuppressive phenotype. Interestingly, the cellular content in different T, B, NK, and myeloid cells was not modified, whereas HLA-DR expression was strongly reduced on 
A

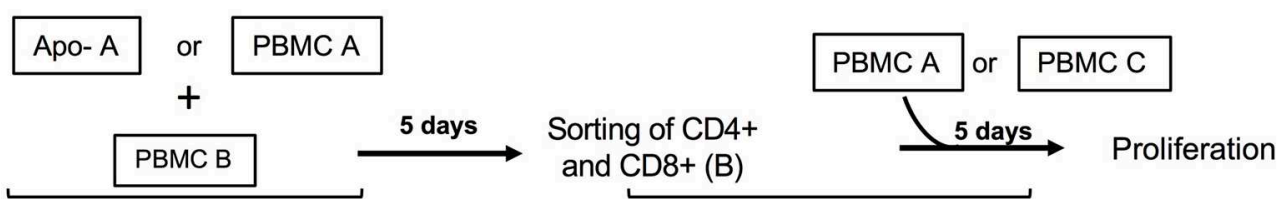

First stim.

Second stim.
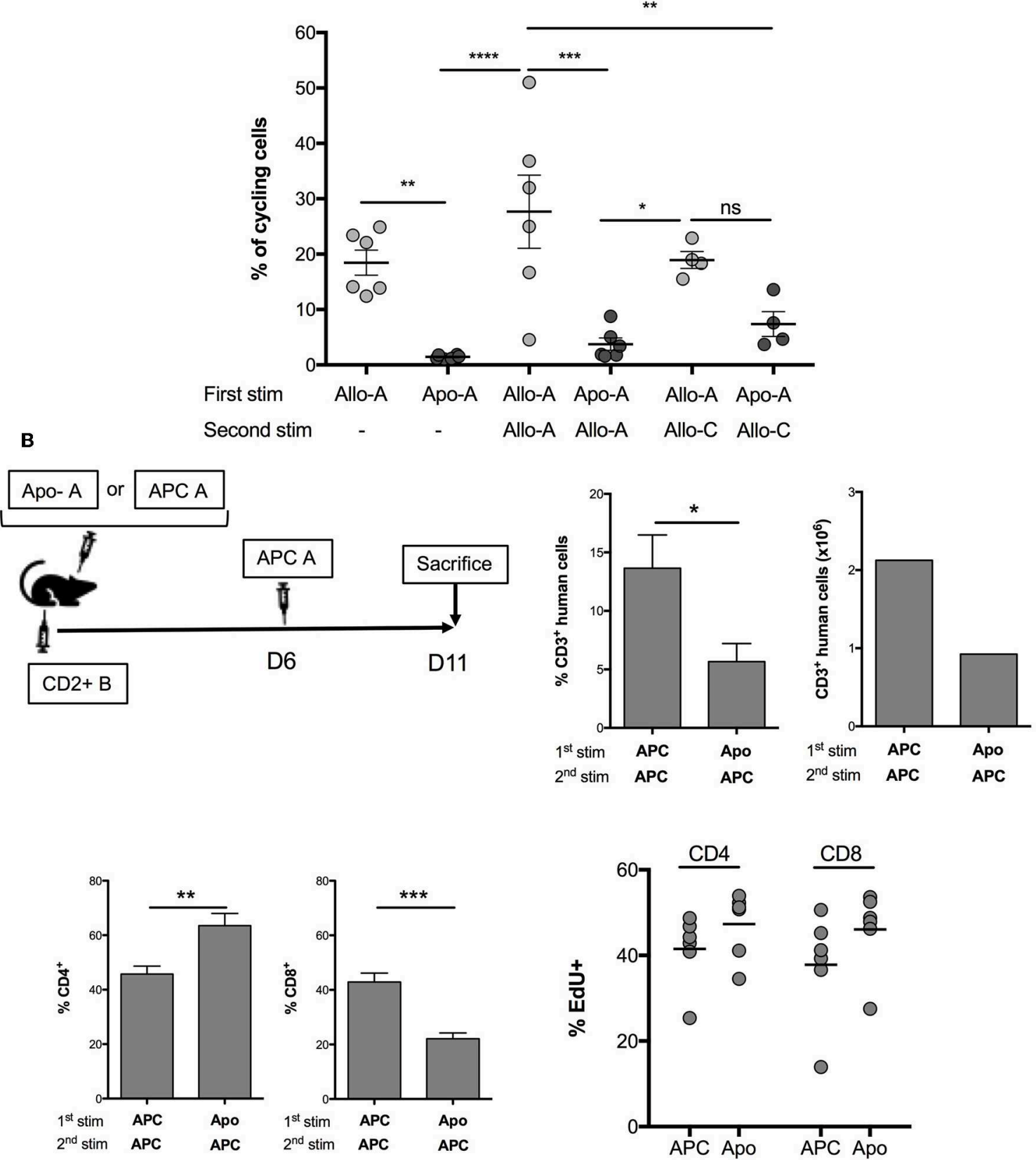

FIGURE 5 | Apo-cells as a tool to modulate allogeneic immune response. (A) Experimental scheme. PBMCs were cultured for 5 days in the presence of either allogeneic Apo-cells or irradiated PBMCs. At day 5, T cells were sorted out by flow cytometry, CFSE-labeled, and cultured in the presence of allogeneic irradiated PBMCs from the same donor or from third-party donor. Cumulative individual and mean \pm SEM data from six independent experiments. Statistics: one-way ANOVA with post-hoc $\left({ }^{*} p<0.05,{ }^{\star \star} p<0.01,{ }^{\star \star *} p<0.001,{ }^{\star \star \star *} p<0.0001\right)$. (B) In vivo, co-injection in NOD/SCID/ $\gamma \mathrm{C}$ (NSG) mice of either allogeneic CD2- cells (APC) or allogeneic Apo-cells with CD2+ cells (containing mainly T and NK cells). Five days later, mice received an additional dose of allogeneic CD2- (APC) cells from the same source used for the first stimulation. 5-Ethynyl-2' -deoxyuridine (EdU) was injected at days 10 and 11 ( $2 \mathrm{~h}$ before sacrifice). Top right, percentages and numbers of human CD3+ cells at day 11 in the spleen of mice (CD3+ in huCD45+). Bottom left, CD4 and CD8 percentages in CD3+ cells, and percentages of EdU+ in CD4 and CD8 cells. Data represent two independent experiments, each with three mice per group. Statistics: unpaired Student $t$-test $\left({ }^{\star} p<0.05,{ }^{* \star} p<0.01,{ }^{\star \star \star} p<0.001\right)$. 
macrophages, DCs, and B cells, similar to the expression of costimulatory CD86 molecule on DCs and B cells. This decreased HLA and CD86 expression could explain the reduced T-cell activation capacity by Apo-cells we observed. The following step, we tried to test the immunosuppressive properties of Apo-cells and to identify the underlying mechanisms. During the rejection process, the allogeneic immune response is initiated primarily by a direct presentation of the donor antigens, hence the importance of demonstrating the suppressive properties of Apo-cells in the direct pathway. Culturing PBMCs in the presence of allogeneic Apo-cells resulted in a strong reduction of responding cells as compared with irradiated PBMCs collected from the same donor of Apo-cells. Not only was the percentage of responding cell proliferation dramatically reduced but also the percentage of activation/memory markers expressed by $\mathrm{T}$ cells. In addition, the concentrations of inflammatory cytokines such as IFN $\gamma$, IL-6, or TNF- $\alpha$ were strongly reduced.

In transplantation, antigen presentation could also result from donor alloantigen processing by recipient APCs and their presentation to autologous responding $\mathrm{T}$ cells. This process is called indirect presentation pathway. We observed that allogeneic Apo-cells had a weak ability to activate APCs (in our model, CD2- cells), as reflected by the reduced HLA-DR expression, and showed a decrease in intensity of expression of co-stimulatory molecules and a decrease in IL-6 and TNF$\alpha$ production. Finally, we aimed at testing the possibility for Apo-cells to suppress alloreactive immune response generated by an indirect presentation pathway both in vitro and in vivo. For such, after a first phase of 5-day in vitro incubation of PBMCs with allogeneic Apo-cells, CD4 and CD8 cells were sorted out and directly restimulated with allogeneic PBMCs from the same donor of Apo-cells. The percentage of cycling T cells was close to zero even after recall stimulation. Importantly, the application of such a tolerance induction protocol by Apo-cells in vivo (in NSG mice) helped us observe a decreased T-cell expansion in vivo.

In summary, this work allowed us to define an Apo-cell generation protocol that is compatible with clinical practice. The obtained Apo-cells and their immunosuppressive properties were phenotypically and functionally characterized in vitro and in vivo. We demonstrate for the first time that Apo-cells can inhibit allogeneic immune response that follows both direct and indirect alloantigen presentation. These results brought the rationale for the use of Apo-cells in tolerance induction protocol

\section{REFERENCES}

1. Edelson R, Berger C, Gasparro F, Jegasothy B, Heald P, Wintroub B, et al. Treatment of cutaneous T-cell lymphoma by extracorporeal photochemotherapy. Preliminary results. $N$ Engl J Med. (1987) 316:297-303. doi: 10.1056/NEJM198702053160603

2. McKenna KE, Whittaker S, Rhodes LE, Taylor P, Lloyd J, Ibbotson S, et al. Evidence-based practice of photopheresis 1987-2001: a report of a workshop of the British Photodermatology Group and the U.K. Skin Lymphoma Group. Br J Dermatol. (2006) 154:7-20. doi: 10.1111/j.1365-2133.2005.06857.x

3. Marshall SR. Technology insight: ECP for the treatment of GvHD-can we offer selective immune control without generalized immunosuppression? Nat Clin Pract Oncol. (2006) 3:302-14. doi: 10.1038/ncponc0511 in organ transplantation. They also provide solid evidence for the registration of an early-phase clinical trial.

\section{DATA AVAILABILITY STATEMENT}

The datasets generated for this study are available on request to the corresponding author.

\section{ETHICS STATEMENT}

Ethical review and approval was not required for the study on human participants in accordance with the local legislation and institutional requirements. The patients/participants provided their written informed consent to participate in this study. The animal study was reviewed and approved by Ethics Committee for Animal Experimentation Charles Darwin (Ce5/2012/025).

\section{AUTHOR CONTRIBUTIONS}

CP, PG, HR, FP, PL, and JC designed the experiment. CP, TS, $\mathrm{AB}-\mathrm{F}, \mathrm{AT}, \mathrm{AB}$, and $\mathrm{CG}$ performed the experiments. $\mathrm{CP}$, TS, $\mathrm{BB}, \mathrm{MM}$, and $\mathrm{GM}$ analyzed the data. $\mathrm{CP}$, JC, and PG wrote the manuscript.

\section{FUNDING}

This work was partly funded by Macopharma and by a dedicated COSSEC grant from INSERM. The funder was not involved in the study design, collection, analysis, interpretation of data, the writing of this article or the decision to submit it for publication.

\section{ACKNOWLEDGMENTS}

We want to thank Marie Tamagne and Jean-Marie Segier from Etablissement Français $d u$ sang for their valuable work on irradiation and UV treatment of human cells, and Aurélie Guguin and Adeline Henry from IMRB for cell sorting.

\section{SUPPLEMENTARY MATERIAL}

The Supplementary Material for this article can be found online at: https://www.frontiersin.org/articles/10.3389/fimmu. 2019.02908/full\#supplementary-material
4. Flowers ME, Apperley JF, van Besien K, Elmaagacli A, Grigg A, Reddy V, et al. A multicenter prospective phase 2 randomized study of extracorporeal photopheresis for treatment of chronic graft-versus-host disease. Blood. (2008) 112:2667-74. doi: 10.1182/blood-2008-03-141481

5. Barr ML, Meiser BM, Eisen HJ, Roberts RF, Livi U, Dall'Amico R, et al. Photopheresis for the prevention of rejection in cardiac transplantation. Photopheresis Transplantation Study Group. N Engl J Med. (1998) 339:174451. doi: 10.1056/NEJM199812103392404

6. Mevorach D, Zuckerman T, Reiner I, Shimoni A, Samuel S, Nagler A, et al. Single infusion of donor mononuclear early apoptotic cells as prophylaxis for graft-versus-host disease in myeloablative HLA-matched allogeneic bone marrow transplantation: a phase I/IIa clinical trial. Biol Blood Marrow Transplant. (2014) 20:58-65. doi: 10.1016/j.bbmt.2013.10.010 
7. Belizaire R, Kim HT, Poryanda SJ, Mirkovic NV, Hipolito E, Savage WJ, et al. Efficacy and immunologic effects of extracorporeal photopheresis plus interleukin-2 in chronic graft-versus-host disease. Blood Adv. (2019) 3:96979. doi: 10.1182/bloodadvances.2018029124

8. Yakut E, Jakobs C, Peric A, Michel G, Baal N, Bein G, et al. Extracorporeal photopheresis promotes IL-1beta production. J Immunol. (2015) 194:256977. doi: 10.4049/jimmunol.1400694

9. Hannani D, Gabert F, Laurin D, Sall M, Molens JP, Hequet O, et al. Photochemotherapy induces the apoptosis of monocytes without impairing their function. Transplantation. (2010) 89:492-9. doi: 10.1097/TP.0b013e3181c6ffd3

10. Fadok VA, Bratton DL, Konowal A, Freed PW, Westcott JY, Henson PM. Macrophages that have ingested apoptotic cells in vitro inhibit proinflammatory cytokine production through autocrine/paracrine mechanisms involving TGF-beta, PGE2, and PAF. J Clin Invest. (1998) 101:890-8. doi: 10.1172/JCI1112

11. Krispin A, Bledi Y, Atallah M, Trahtemberg U, Verbovetski I, Nahari E, et al. Apoptotic cell thrombospondin-1 and heparin-binding domain lead to dendritic-cell phagocytic and tolerizing states. Blood. (2006) 108:35809. doi: 10.1182/blood-2006-03-013334

12. Schmitt DA, Ullrich SE. Exposure to ultraviolet radiation causes dendritic cells/macrophages to secrete immune-suppressive IL-12p40 homodimers. $J$ Immunol. (2000) 165:3162-7. doi: 10.4049/jimmunol.165.6.3162

13. Morelli AE, Larregina AT, Shufesky WJ, Zahorchak AF, Logar AJ, Papworth GD, et al. Internalization of circulating apoptotic cells by splenic marginal zone dendritic cells: dependence on complement receptors and effect on cytokine production. Blood. (2003) 101:611-20. doi: 10.1182/blood-2002-06-1769

14. Ren G, Su J, Zhao X, Zhang L, Zhang J, Roberts AI, et al. Apoptotic cells induce immunosuppression through dendritic cells: critical roles of IFN-gamma and nitric oxide. J Immunol. (2008) 181:3277-84. doi: 10.4049/jimmunol.181.5.3277

15. Sauter B, Albert ML, Francisco L, Larsson M, Somersan S, Bhardwaj N. Consequences of cell death: exposure to necrotic tumor cells, but not primary tissue cells or apoptotic cells, induces the maturation of immunostimulatory dendritic cells. J Exp Med. (2000) 191:423-34. doi: 10.1084/jem.191.3.423

16. Albert ML, Sauter B, Bhardwaj N. Dendritic cells acquire antigen from apoptotic cells and induce class I-restricted CTLs. Nature. (1998) 392:869. doi: $10.1038 / 32183$

17. Steinman RM, Nussenzweig MC. Avoiding horror autotoxicus: the importance of dendritic cells in peripheral $\mathrm{T}$ cell tolerance. Proc Natl Acad Sci USA. (2002) 99:351-8. doi: 10.1073/pnas.231606698

18. Wang Z, Larregina AT, Shufesky WJ, Perone MJ, Montecalvo A, Zahorchak AF, et al. Use of the inhibitory effect of apoptotic cells on dendritic cells for graft survival via T-cell deletion and regulatory T cells. Am J Transplant. (2006) 6:1297-311. doi: 10.1111/j.1600-6143.2006.01308.x

19. Coppard C, Hannani D, Humbert M, Gauthier V, Plumas J, Merlin E, et al. In vitro PUVA treatment triggers calreticulin exposition and HMGB1 release by dying T lymphocytes in GVHD: new insights in extracorporeal photopheresis. J Clin Apher. (2019) 34:450-60. doi: 10.1002/jca.21698

20. Sabbagh L, Bourbonniere M, Sekaly RP, Cohen LY. Selective up-regulation of caspase-3 gene expression following TCR engagement. Mol Immunol. (2005) 42:1345-54. doi: 10.1016/j.molimm.2004.12.011

21. Burlion A, Brunel S, Petit NY, Olive D, Marodon G. Targeting the human Tcell inducible COStimulator molecule with a monoclonal antibody prevents graft-vs-host disease and preserves graft vs leukemia in a xenograft murine model. Front Immunol. (2017) 8:756. doi: 10.3389/fimmu.2017.00756

22. Cherai M, Hamel Y, Baillou C, Touil S, Guillot-Delost M, Charlotte F, et al. Generation of human alloantigen-specific regulatory $\mathrm{T}$ cells under good manufacturing practice-compliant conditions for cell therapy. Cell Transplant. (2015) 24:2527-40. doi: 10.3727/096368914X683566

23. Touil S, Rosenzwajg M, Landau DA, Le Corvoisier P, Frederic C, Klatzmann D, et al. Depletion of $\mathrm{T}$ regulatory cells through selection of CD127-positive cells results in a population enriched in memory $\mathrm{T}$ cells: implications for anti-tumor cell therapy. Haematologica. (2012) 97:167885. doi: 10.3324/haematol.2011.056374

24. Vallion R, Bonnefoy F, Daoui A, Vieille L, Tiberghien P, Saas $\mathrm{P}$, et al. Transforming growth factor-beta released by apoptotic white blood cells during red blood cell storage promotes transfusion-induced alloimmunomodulation. Transfusion. (2015) 55:1721-35. doi: 10.1111/trf.13031

25. Bittencourt MC, Perruche S, Contassot E, Fresnay S, Baron MH, Angonin R, et al. Intravenous injection of apoptotic leukocytes enhances bone marrow engraftment across major histocompatibility barriers. Blood. (2001) 98:22430. doi: 10.1182/blood.V98.1.224

26. Chen W, Jin W, Tian H, Sicurello P, Frank M, Orenstein JM, et al. Requirement for transforming growth factor betal in controlling $\mathrm{T}$ cell apoptosis. J Exp Med. (2001) 194:439-53. doi: 10.1084/jem.194.4.439

27. Gao Y, Herndon JM, Zhang H, Griffith TS, Ferguson TA. Antiinflammatory effects of CD95 ligand (FasL)-induced apoptosis. J Exp Med. (1998) 188:88796. doi: 10.1084/jem.188.5.887

28. Huang FP, Platt N, Wykes M, Major JR, Powell TJ, Jenkins CD, et al. A discrete subpopulation of dendritic cells transports apoptotic intestinal epithelial cells to T cell areas of mesenteric lymph nodes. J Exp Med. (2000) 191:435-44. doi: 10.1084/jem.191.3.435

29. Kleinclauss F, Perruche S, Masson E, de Carvalho Bittencourt M, Biichle S, Remy-Martin JP, et al. Intravenous apoptotic spleen cell infusion induces a TGF-beta-dependent regulatory T-cell expansion. Cell Death Differ. (2006) 13:41-52. doi: 10.1038/sj.cdd.44 01699

30. Xia CQ, Peng R, Qiu Y, Annamalai M, Gordon D, Clare-Salzler MJ. Transfusion of apoptotic beta-cells induces immune tolerance to beta-cell antigens and prevents type 1 diabetes in NOD mice. Diabetes. (2007) 56:211623. doi: $10.2337 / \mathrm{db} 06-0825$

31. Kim S, Elkon KB, Ma X. Transcriptional suppression of interleukin-12 gene expression following phagocytosis of apoptotic cells. Immunity. (2004) 21:643-53. doi: 10.1016/j.immuni.2004.09.009

Conflict of Interest: The authors declare that the research was conducted in the absence of any commercial or financial relationships that could be construed as a potential conflict of interest.

Copyright (c) 2019 Pilon, Stehlé, Beldi-Ferchiou, Matignon, Thiolat, Burlion, Grondin, Birebent, Pirenne, Rouard, Lang, Marodon, Grimbert and Cohen. This is an open-access article distributed under the terms of the Creative Commons Attribution License (CC BY). The use, distribution or reproduction in other forums is permitted, provided the original author(s) and the copyright owner(s) are credited and that the original publication in this journal is cited, in accordance with accepted academic practice. No use, distribution or reproduction is permitted which does not comply with these terms. 